medium-temperature tempering. High speed of cooling under getting of not big castings or the castings with wall thickness to $25-30 \mathrm{~mm}$ allow to get at once austenite-martensite structure. In other cases, the loading into furnace after hardening of casting at temperature $950^{\circ} \mathrm{C}$, endurance $2-3$ hours and cooling together with furnace or hardening in an open air is used. The probability of graphitization of castings under synthesis of alloy by aluminothermic way decreases considerably because of considerable gradient of temperatures and high speed of heat abstraction, i.e. getting of martensite structure under casting goes considerably simpler.

Thus we may make a conclusion that aluminothermic ways can be used for producing of special thermite alloyed cast irons expect for high-chromium cast irons during the synthesis of those the problems of technological character appear. Other types of special cast irons have in some cases even better properties than in cast irons produced by ordinary methods. Designed compositions of thermite mixtures are also suitable for technology of thermite casting additives of high-temperature gradient.

\title{
References
}

1. Zhiguts Yu., Lazar V. 2014. Special grey and white termite cast irons .British Journal of Science, Education and Culture, "London University Press”. London. № 2 (6). V. 1. P. 201 - 207.

2. Zhiguts Yu.Yu., Opathko I.I. 2014. The highstrenght thermite cast irons. American Journal of Scientific and Educational Research, "Columbia Press". New York. № 2 (5). V. 2. P. 635 $-641$.

3. Zhiguts Yu.Yu., Homjak B.Ya., Bilak I.V. 2015. Synthesis of special thermite cast irons and their properties. Fundamental and applied science: 11 international research and practice konf., 30 October - 07 November 2015 r.: materials conf. Sheffield UK: "Science and Education" LTD. V. 18. P. 30 - 32.

4. Zhiguts Yu., Beiresh Ya. 2017. The alloyed cast irons synthesized by metallothermic processes. Conduct of modern science: 13 international research and practical konf.: 30 November 07 December 2017 y.: materials conf. Technical science. Sheffield UK: "Science and Education" LTD. V. 13. P. 14 - 16.

УДК 662.767.1=161.1

\section{СФЕРИЧЕСКИЙ ОПТОЭЛЕКТРОННЫЙ СЕНСОР ДЛЯ ИЗМЕРЕНИЯ КОНЦЕНТРАЦИИ МЕТАНА В ВОЗДУХЕ}

Кабаций В. Н., Хомяк Б.Я., Питьовка О.Ю.

\section{SPHERICAL OPTOELECTRONIC SENSOR FOR MEASUREMENT OF METHANE CONCENTRATION IN AIR}

Kabatsiy Vasyl, Khomyak Bogdan, Pityovka Oksana

Предложена конструкция оптоэлектронного сенсора газа на метан $\left(\mathrm{CH}_{4}\right)$ учитывающая особенности спектральных характеристик источников и приемников среднего ИК-диапазона спектра. Показана возможность использования оптоэлектронного сенсора для измерения конщентращии $\mathrm{CH}_{4}$ в диапазоне 0-3 об.\%. Минимально измеренная концентрачия газа в воздухе ограничивается только отношением сигнал/шум и составляет 200-250 ppm.

Ключевые слова: ИК-излучения, оптоэлектронные сенсоры, фотоприемники, оптоэлектронный сенсор, газ. 
Proposed the design of an optoelectronic gas sensor for methane $\left(\mathrm{CH}_{4}\right)$ takes into account the features of the spectral characteristics of sources and receivers of the middle IR spectral range. The possibility of using an optoelectronic sensor for measuring the concentration of $\mathrm{CH} 4$ in the range 0-3 vol.\% has shown. The minimum measured gas concentration in air is limited only by the signal/noise ratio and is 200-250 ppm.

Keywords: IR radiation, optoelectronic sensors, photodetectors, gas, optoelectronic sensor.

Актуальной задачей в области контроля технологических процессов в промышленном производстве и теплоэнергетике, экологического контроля выбросов загрязняющих газов в атмосферу является измерение концентрации молекулярных компонентов газовой смеси. Загрязняющие атмосферу газы образуются также при выхлопах автомобиля и неполном сгорании топлива в печи или камине, выделяются из современных отделочных материалов и упаковок при их тлении или горении, а также на начальной стадии пожара при пиролизе или нестандартных режимах функционирования электрооборудования. Эффективно оценить состояние атмосферы и концентрации в ней вредных газов возможно только при условиях объективного контроля инструментальными средствами.

В последние годы наблюдается переход от традиционных средств анализа газа, в котором используются лампы накаливания, оптические фильтры и механические модуляторы излучения, к использованию нового поколения многофункциональных приборов газового анализа, разработанных и изготовленных на современной элементной оптоэлектронной базе [1]. Учитывая, что основными элементами современного оптоэлектронного сенсора являются полупроводниковые источники и фотоприемники ИКизлучения, то для достижения высокого кпд сенсора необходимо, чтобы параметры его составных частей были согласованы по спектральным характеристикам, быстродействию, температурным свойствам и габаритам. Помимо этого, источники ИК-излучения, применяемые в оптоэлектронных сенсорах, должны обладать достаточно узкой направленностью излучения и работать при сравнительно небольших входных токах. Поэтому совершенствованию полупроводниковых источников ИК-излучения уделяется большое внимание [2-5]. Не менее важным является и выбор фотоприемника, который должен обладать не только высокой эффективностью преобразования падающего на него излучения в электрический ток, но и требуемым спектральным распределением фоточувствительности и быстродействию [3].

Использование полупроводниковых источников и фотоприемников ИК-излучения существенно повышает чувствительность, селективность, быстродействие, экономичность и надежность спектроабсорбционных приборов анализа составов газовых смесей, значительно уменьшает габариты и материалоемкость, а также позволяет автоматизировать процесс измерения, применять непрерывный контроль состояния окружающей среды и промышленных процессов [1-4].

Настоящая работа посвящена разработке оптоэлектронных сенсоров газов на метан и двуокись углерода с использованием многоэлементных полупроводниковых источников излучения и фотоприемников, работающих в спектральном диапазоне 2,5-5,0 мкм.

1. Многоэлементные полупроводниковые источники ИК-излучения на область спектра 2,5-5,0 мкм.

Излучающие активные элементы (АЭ), с $p$-n-переходами и высокими коэффициентами инжекции при электролюминисценции, полупроводниковых источников излучения работающих при комнатной температуре, изготовлены на базе твердых растворов (TP) InGaAs/InAs и InAsSbP/InAs методом жидкостной эпитаксии, позволяющим выращивать на монокристаллической подложке высококачественные гетероструктуры [2]. Для повышения внешнего квантового выхода на излучающие АЭ наносились оптические покрытия, одновременно выполняющие механическую защиту, эффект просветления и 
фокусирование излучения. В качестве материалов для оптического покрытия, использовались халькогенидные стеклообразные полупроводники (ХCП) на базе многокомпонентных систем $\mathrm{Ge}(\mathrm{Pb})-\mathrm{Sb}(\mathrm{Bi}, \mathrm{Ga})-\mathrm{S}(\mathrm{Se})[6]$.

Оптическое покрытие из ХСП в виде вытянутой полусферы сужает диаграмму направленности излучения вдоль оптической оси от $160^{0}$ до $40^{0}$ и повышает мощность излучения АЭ в 2,5-3,0 раза. При использовании оптического покрытия в виде цилиндрической поверхности с полусферическим куполом удалось сузить диаграмму направленности излучения до $15^{0}$ и увеличить мощность излучения в 3,5-4 раза [5]. Разработанная нами технология нанесения оптического покрытия на излучающие АЭ обеспечивает удовлетворительную воспроизводимость геометрии стеклянного купола [7].

Конструкции полупроводниковых источников излучения [2, 6-9] разработаны таким образом, что позволяют разместить несколько АЭ на одной теплопроводной основе и могут излучать на одной или разных длинах волн, а их питание осуществляется раздельно в непрерывном или импульсном режиме. Комплексные исследования электрофизических и оптических параметров активных элементов позволили определить оптимальные составы ТР, обеспечивающие излучение АЭ на длинах волн, согласованных с длиной волны собственного поглощения соответствующего газа или компонентов в газовой смеси (рис. 1) (длины волн излучения 2,9 мкм, 3,32 мкм, 4,27 мкм, 4,67 мкм отвечают полосам поглощения паров воды, метана $\left(\mathrm{CH}_{4}\right)$, углекислого газа $\left(\mathrm{CO}_{2}\right)$, окиси углерода $\left.(\mathrm{CO})\right)$.

Вследствие изменения ширины запрещенной зоны материалов $\mathrm{A}^{3} \mathrm{~B}^{5}$ с повышением температуры внешней среды спектральные характеристики излучающих АЭ, изготовленных на их основе, смещаются в длинноволновую область спектра. Изменение температуры АЭ происходит также вследствие разогрева $p-n$-перехода при увеличении прямого тока через него. Поэтому, при проектировании и изготовлении оптоэлектронных сенсоров газа необходимо знать и учитывать температурный коэффициент смещения максимума в спектре излучения АЭ. Спектральные характеристики АЭ измерялись в диапазоне температур 250330 К. На основании этих измерений для АЭ, используемых нами в оптоэлектронном сенсоре на метан, представлены рис. 3 температурные зависимости спектрального положения максимума в спектре излучения и полуширины спектра ( $\mathrm{I}=200 \mathrm{MA}, v=10^{3}$ Гц, $\tau=500$ мкс).

І, отн.ед.

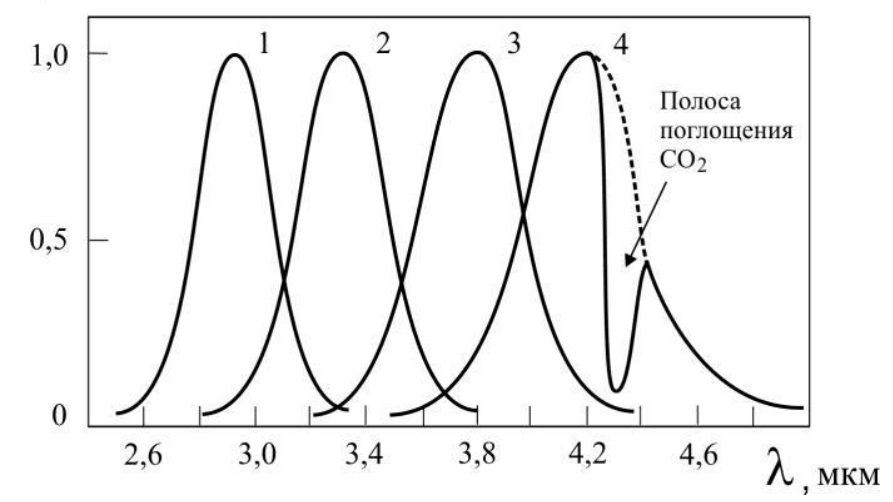

Рис. 1. Зависимости интенсивности излучения $\boldsymbol{p}-\boldsymbol{n}$-перехода АЭ от длины волны излучения при $\mathbf{T}=\mathbf{3 0 0} \mathrm{K}$, согласованные с максимумами длин волн собственного поглощения различных газов: 1 - паров воды $\left(\lambda=2,9\right.$ мкм); 2 - метана $\left(\mathrm{CH}_{4}, \lambda=3,32\right.$ мкм); $3-\lambda=3,9$ мкм (используется для опорного канала в оптоэлектронном сенсоре); 4двуокиси углерода ( $\lambda==4,27$ мкм). 


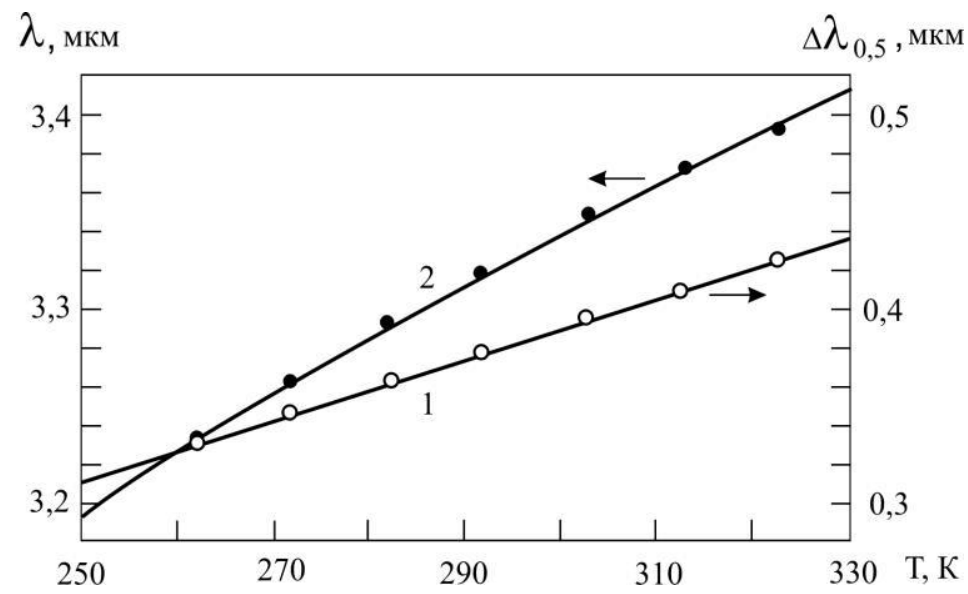

Рис. 2. Температурные зависимости спектрального положения максимума в спектре излучения (1) и полуширины спектра (2) излучения АЭ, используемого в оптоэлектронном сенсоре на метан.

Для измерения мощности излучения АЭ в области спектра 2,5-5,0 мкм использовалась интегрирующая сфера и калибровочный охлаждаемый $\mathrm{PbSe}$ фотоприемник. Внутренняя поверхность сферы диаметром 80 мм покрывалась мятой алюминиевой фольгой с коэффициентом диффузного отражения 0,9-0,92. Источник ИК-излучения и ФП расположены на противоположных сторонах сферы и разделены между собой диффузно рассеивающим экраном. Используя свойства интегрирующей сферы, мощность излучения рассчитывалась по формуле:

$$
P=\frac{4 D^{2}(1-\beta) U}{d^{2} S p},
$$

где $D$ - внутренний диаметр сферы, м; $\beta$ - коэффициент диффузного отражения поверхности сферы; $U$-электрический сигнал на выходе с $\Phi П, \mathrm{~B} ; d$-диаметр чувствительной площадки ФП, м; $S_{p}$ - интегральная чувствительной ФП по отношению к исследуемому источнику излучения, В/Вт.

До начала измерений интегрирующая сфера продувалась осушенным азотом в десятикратном объеме от объема сферы. Ошибка измерений мощности излучения АЭ не превышала 15 \%. Мощность излучения АЭ измерялась в диапазоне температур 250-330 К и плотности $(J)$ прямого тока $(I)$, проходящего через АЭ от $1 \mathrm{~A} / \mathrm{cm}^{2}$ до $10^{3} \mathrm{~A} / \mathrm{cm}^{2}$. Длительность $(\tau)$ прямоугольных импульсов изменялась от 25 мкс до 500 мкс при частоте $(v)$ их следования от 10 до $10^{6}$ Гц. Для всех исследуемых АЭ мощность излучения уменьшается с повышением температуры рис. 4 ( $\mathrm{I}=200 \mathrm{MA}, v=10^{3} \Gamma ц, \tau=500$ мкс).

На рис. 4 приведены зависимости мощности излучения АЭ, излучающих на различных длинах волн, от величины прямого тока через $p-n$-переход $\left(\mathrm{T}=293 \mathrm{~K}, \nu=10^{3} \Gamma ц, \tau=500\right.$ мкс). Из этого рисунка видно, что увеличение прямого тока через $p-n$-переход приводит к увеличению мощности излучения для всех исследуемых АЭ вплоть до тока 250 мА $\left(25 \mathrm{~A} / \mathrm{cm}^{2}\right)$, достигая насыщения, после чего мощность излучения АЭ начинает уменьшаться с увеличением тока больше 250 мА $\left(25 \mathrm{~A} / \mathrm{cm}^{2}\right)$, вследствие нагревания $p$ - $n$-перехода. Изменение режима работы АЭ ( $v=10$ Гц, $\tau=25-50$ мкс) приводит к тому, что $p-n$-переход не успевает нагреться даже при токе 7А $\left(700 \mathrm{~A} / \mathrm{cm}^{2}\right)$, а мощность излучения возрастает в несколько раз. Увеличение плотности тока через $p$-n-переход приводит к смещению максимума в спектрах излучения АЭ в длинноволновую область и увеличению полуширины спектров. Так для активных элементов, изготовленных на базе TP InGaAs (область излучения 2,8-3,6 мкм), смещение максимума излучения и увеличение полуширины спектров излучения наблюдается при плотности тока $25 \mathrm{~A} / \mathrm{cm}^{2}$. Для активных элементов, 
изготовленных на базе TP InAsSbP (область излучения 3,8-5,0 мкм), такие изменения наблюдаются уже при плотности тока $10 \mathrm{~A} / \mathrm{cm}^{2}$.

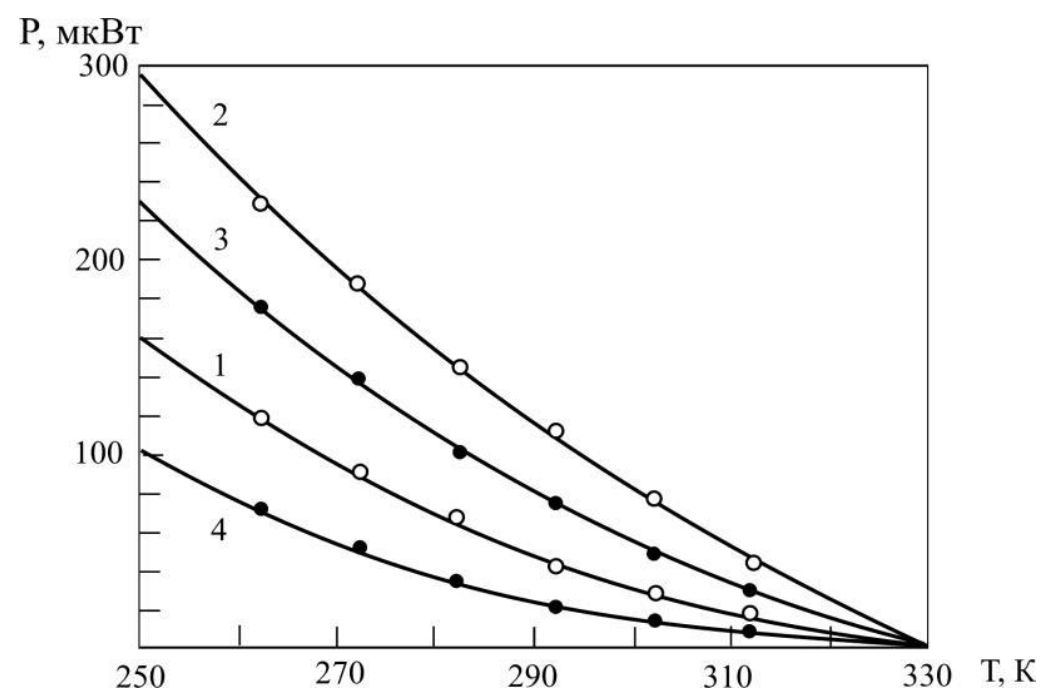

Рис. 3. Температурные зависимости мощности излучения различных АЭ, излучающих на длинах волн в максимуме излучения $\lambda_{\max }$ 1- 2,9 мкм; $2-3,32$ мкм; 3-

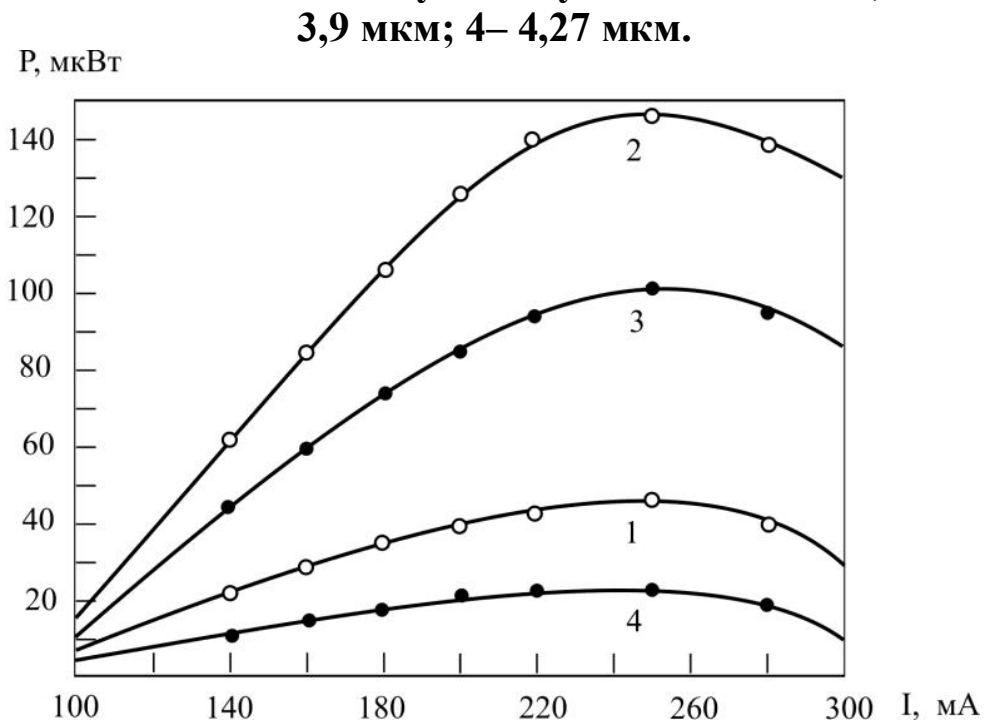

Рис. 4. Зависимости мощности излучения АЭ от прямого тока через $p-n$-переход на различных длинах волн в максимуме излучения $\lambda_{\max }: 1-2,9$ мкм; 2- 3,32 мкм; 3- 3,9 мкм; 4- 4,27 мкм.

Таким образом, приведенные выше результаты указывают на необходимость температурной стабилизации активных элементов при использовании их в оптоэлектронных сенсорах газов. Использование различных типов полупроводниковых микрохолодильников, работающих на эффекте Пэльтье, позволяет стабилизировать спектральное положение максимума в спектре излучения АЭ, а также увеличить на 20 \% мощность излучения, за счет увеличения прямого тока через $p-n$-переход. Вместе с тем следует отметить, что использование таких микрохолодильников существенно увеличивает потребляемую мощность источника излучения, усложняет его конструкцию и уменьшает надежность. В то же время, используя указанное поведение мощности излучения и спектральных характеристик АЭ, возникает возможность управление этими параметрами с помощью прямого тока через $p-n$-переход [2].

2. Фотоприемники ИК-излучения на область спектра 2,5-5,0 мкм. 
Приемники оптического излучения являются неотъемлемым элементом современной аппаратуры для оптической обработки информации. Основным условием использования параметров фотоприемников в составе сложных оптоэлектронных устройств есть правильный выбор типа приемника излучения. В нашем случае, учитывая электрические и оптические характеристики разработанных источников ИК-излучения, для обеспечения надежного приема и обработки электрического сигнала необходим ФП с интегральной вольтовой чувствительностью не менее $10^{3} \mathrm{~B} / \mathrm{BT}$ и обнаружительной способностью не менее $10^{9} \mathrm{~cm} \cdot \Gamma_{ц}{ }^{1 / 2} \cdot \mathrm{BT}^{-1}$ по АЧТ при $T=300$ К. Спектральные характеристики различных типов ФП, на область спектра 2,5-5,0 мкм, приведены на рис.5.

Важной характеристикой ФП является постоянная времени, определяющая верхнюю границу частоты модуляции излучения, превышение которой приводит к снижению интегральной чувствительности ФП. При выборе ФП необходимо также учитывать размер фоточувствительной площадки, поскольку граничная чувствительность однотипных ФП зависит от ее размеров и ухудшается с ее увеличением. В такой же зависимости от размеров чувствительной площадки находятся и шумы ФП. Однако этот шум уменьшается при использовании модулированного сигнала с усреднением.

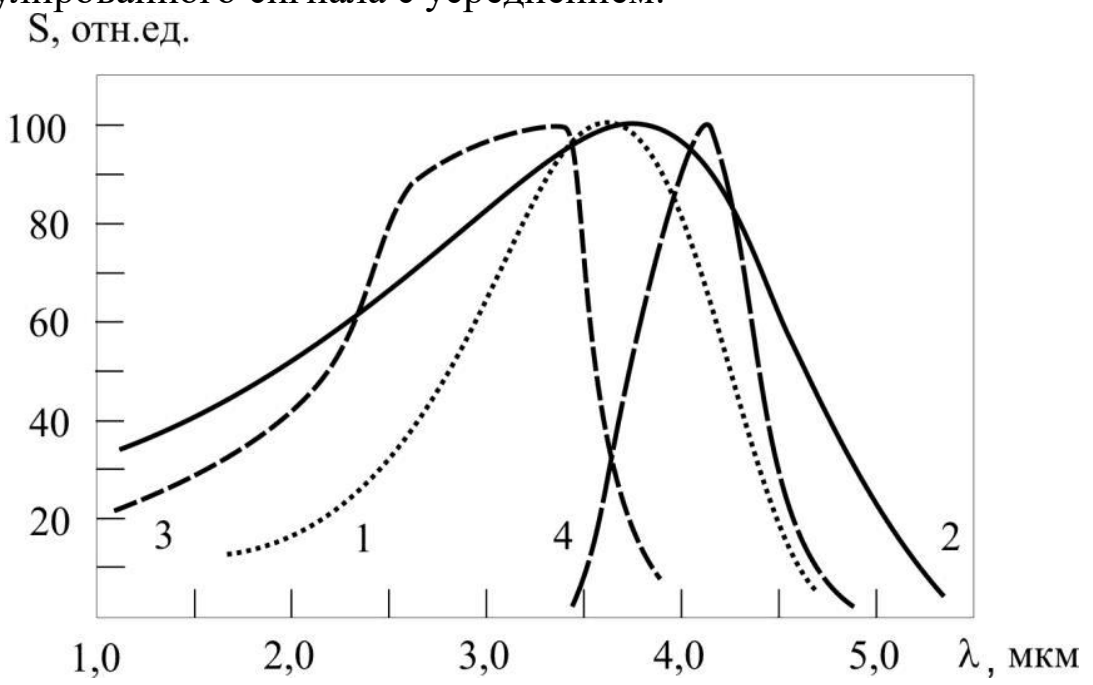

Рис. 5. Спектральные характеристики различных ФП в средней ИК-области спектра:

1- PbSe фоторезистор; 2-охлаждаемый PbSe фоторезистор; 3- InAs фотодиод [3]; 4- фотодиод PD42Sr фирмы MIRDOG (PФ).

Так как, использование модулированного излучения с частотой 500 Гц и усреднением больше 1 с, приводит к увеличению соотношения сигнал/шум до 20 раз, а при 10 с до 70 раз [3]. Такое время усреднения вполне приемлемо для большинства газоанализаторов, так как изменение величины концентрации газа происходит за время больше $1 \mathrm{c} . \mathrm{PbSe}$ фоторезисторы характеризуются достаточной чувствительностью в области спектра 2,5-5,0 мкм и их наиболее часто используют в приборах анализа концентрации газа.

C учетом конструктивных и технических особенностей, разработанных нами многоэлементных источников ИК-излучения $[2,7,8]$ и характеристик ФП нами разработан оптоэлектронный сенсор для измерения концентрации $\mathrm{CH}_{4}$ в газовой смеси.

3. Оптоэлектронный сенсор на метан $\left(\mathrm{CH}_{4}\right)$

Для измерения концентрации $\mathrm{CH}_{4}$ в диапазоне $0-3,0$ об.\% с коэффициентом поглощения $\alpha_{\max }=0,1$ и $\alpha_{\mathrm{eff}}=0,05$ в области 3,3 мкм [3], изготовлен оптоэлектронный сенсор в виде интегрирующей сферы рис. 7 [11].

Оптоэлектронный сенсор состоит из оптически связанных источника ИК-излучения 1 , интегрирующей сферической кюветы 2, светорассеивающего экрана 3 и фотоприйомника 4. Внутренняя поверхность кюветы и основание светоотражающего экрана, покрыты сильно 
деформированной алюминиевой фольгой, толщиной 0,02 мм, создающей диффузное отражение с коэффициентом отражения $0,90-0,92$ в диапазоне длин волн 2,5-5,0 мкм. Светоотражающий экран выполнен в виде многогранной правильной пирамиды с площадью основания в два раза большей площади сечения светового потока, создаваемого источником излучения на входе кюветы. Грани пирамиды зеркальные и направлены в сторону источника излучения.

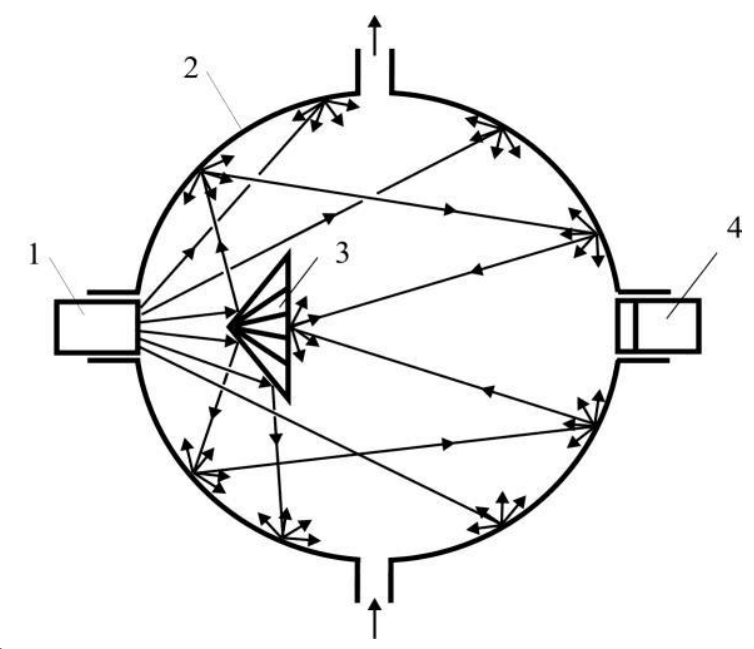

Рис. 6. Конструкция оптоэлектронного сенсора на метан:

1- многоэлементный источник ИК-излучения; 2- интегрирующая сферическая кювета; 3- светоотражающий экран; 4- фотоприемник.

Для обеспечения минимальных потерь на рассеивающей поверхности кюветы, входные и выходные отверстия подачи метана изготовлены в виде отдельных отверстий диаметром 1 мм. Многоэлементный источник ИК-излучения состоит из нескольких АЭ, излучающих на рабочей длине волны $\lambda_{\max 1}=3,32$ мкм $\left(\Delta \lambda_{0,5}=0,38\right.$ мкм) и на длине волны в максимуме излучения $\lambda_{\max 2}=3,9$ мкм $\left(\Delta \lambda_{0,5}=0,42\right.$ мкм), за полосой поглощения метана (опорный канал). Прямая модуляция световых потоков обеспечивается активацией источников ИК-излучения переменным током $I=250$ мА, частотой $v=10^{3}$ Гц и длительностью импульса $\tau=500$ мкс. Регистрация ИК-излучения осуществляется $\mathrm{PbSe}$ фоторезистором. Световой поток от источника излучения 1, попадая внутрь кюветы 2 , отражаясь экраном 3 и рассеиваясь внутренней поверхностью кюветы, создает некоторый уровень освещенности, который регистрируется ФП.

При подаче метана внутрь кюветы, световой поток ослабляется вследствие частичного поглощения. В результате внутри кюветы создается освещенность пропорциональная ослаблению излучения метаном, которая регистрируется ФП. Изменение освещенности внутри кюветы при наличии в ней метана, приводит к соответствующему изменению выходного сигнала ФП, который служит мерой концентрации метана. На рис. 8 приведены зависимости падения напряжения на выходе ФП от концентраций метана в сферической кювете (кривая 1) и цилиндрической кювете длиной 164 мм (кривая 2).

Учитывая относительно большой объем кюветы, оптимальная скорость подачи газовой смеси составляет 2-2,5 л/мин, при этом время установления показаний не превышает 20-30 с. Воспроизводимость показаний при проведении измерений не хуже $\pm 2 \%$, а стабильность измерений при 60-минутном пропускании газовой смеси с концентрацией 0,3 $\%$ составляет $\pm 4 \%$. Отношение сигнал-шум достигает не менее 100. 


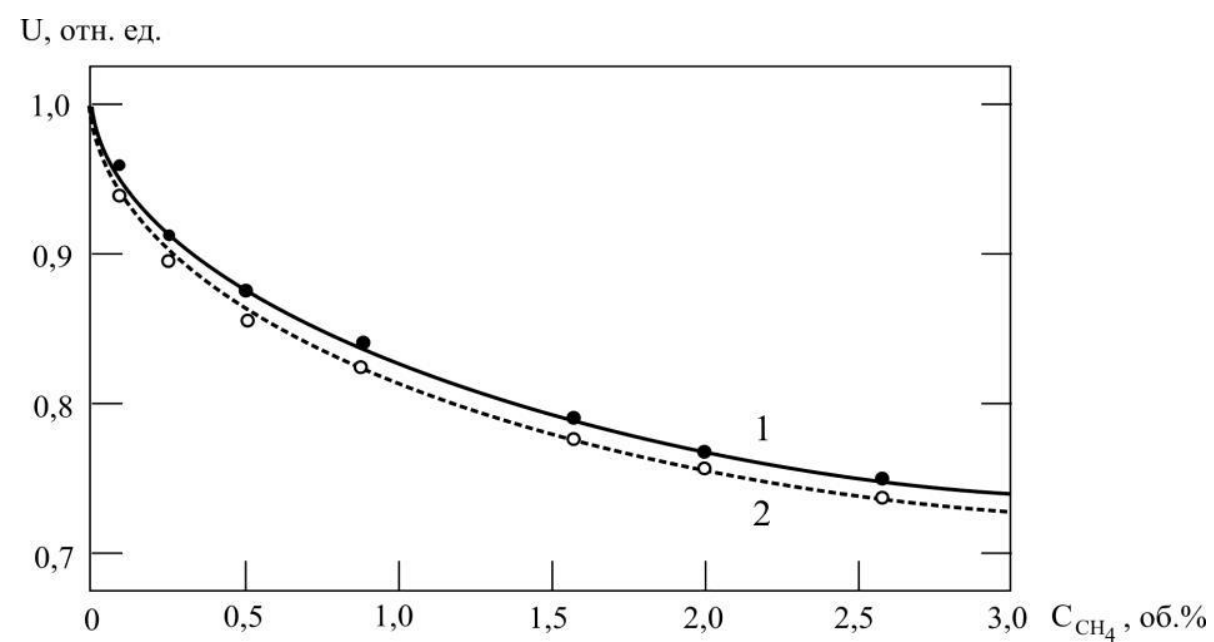
Рис. 7. Зависимость падения напряжения на выходе ФП от концентрации метана (CH4) для сферической кюветы (1) диаметром 80 мм и цилиндрической кюветы (2) длиной 164 мм.

Минимально измеряемая концентрация $\mathrm{CH}_{4}$ в воздухе составляет 200-250 ppm. При использовании сферической кюветы приведенная ошибка измерений составляла 5\%. Сравнительные измерения концентрации $\mathrm{CH}_{4}, \mathrm{c}$ использованием сферических и цилиндрических кювет оптоэлектронного сенсора показали, что сферические кюветы являются более перспективными и позволяют уменьшать габариты и массу сенсора.

Разработанный оптоэлектронный сенсор газа позволяет конструировать современные многофункциональные приборы газового анализа с высокими эксплуатационными параметрами. Компактность, виброустойчивость, малые габариты и масса, вместе с большим сроком службы и быстродействием определяют широкий спектр их использования.

\section{Использованные источники}

1. Александров С.Е. Моделирование характеристик оптических газовых сенсоров на основе диодных оптопар среднего ИК-диапазона спектра / С.Е. Александров, Г.А. Гаврилов, А.А. Капралов, Б.А. Матвеев, Г.Ю. Сотникова, М.А. Ременный // Журнал технической физики. - 2009. - Т.79, № 6. - С. 112-118.

2. Aleksandrov S. Portable optoelectronic gas sensors operating in the mid-IR spectral range $(\lambda=3-5 \mu \mathrm{m})$ / S. Aleksandrov, G. Gavrilov, A. Kapralov, S. Karandashov, B. Matveev, G. Sotnikova, N. Stus // Lasers for Measurements and Information Transfer: Intern. Conf., 2002: Proc. SPIE. - Vol. 4680. - P.188-194.

3. Remennyi M.A. Low voltage episide down bonded mid-IR diode optopairs for gas sensing in the 3,3-4,3 $\mu \mathrm{m}$ spectral range/ M.A. Remennyi, N.V. Zotova, S.A. Karandashev, B.A. Matveev., N.M. Stus', G.N. Talalakin // Sensors \& Actuatoators B: Chemical. - 2003. - Vol. 91. № 1-3. - P. 256-261.

4. Зотова Н. В. Источники спонтанного излучения на основе арсенида индия / Н. В. Зотова, Н. Д. Ильинская, С. А. Карандашёв, Б. А. Матвеев, М. А. Ременный, Н. М. Стусь // Физика и техника полупроводников. - 2008. - Т.42. - № 4. - Р. 641-657.

5. Кабаций В. Н. Объемные оптические покрытия из халькогенидных стекол для полупроводниковых источников ИК-излучения // Технология и конструирование в электронной аппаратуре. - 2009. - № 4. - С. 38-44.

6. Пат. № 88565 Україна. Захисне, просвітлююче та фокусуюче покриття на основі халькогенідного скла і спосіб його нанесення / Блецкан Д.І., Кабацій В.М. - 10.07.2009.

7. Пат. № 8564 Україна. Напівпровідникове джерело інфрачервоного випромінювання / Кабацій В.М. - 10.02.2009. 
8. Пат. № 86900 Україна. Багатоканальне джерело випромінювання (варіанти) / Кабацій В.М., Блецкан Д.І. - 25.05.2009.

9. Пат. № 89707 Україна. Оптичний сенсор газів / Кабацій В.М., Блецкан Д.І., Гасинець В.О. - 25.02.2010.

\section{References}

1. Alexandrov S.E., Gavrilov G.A., Kapralov A.A., Matveev B.A., Sotnikova G.Yu., Remennyi M.A. 2009. Modelyrovanye kharakterystyk optycheskykh hazovukh sensorov na osnove dyodnukh optopar sredneho YK-dyapazona spektra [Modeling of the characteristics of optical gas sensors based on diode optocouplers of the middle IR spectral range]. Journal of Technical Physics. T.79. № 6:112-118.

2. Aleksandrov S., Gavrilov G., Kapralov A., Karandashov S., Matveev B., Sotnikova G., Stus N. 2002. Portable optoelectronic gas sensors operating in the mid-IR spectral range $(\lambda=3-5 \mu \mathrm{m})$ [Portable optoelectronic gas sensors operating in the mid-IR spectral range $(\lambda=3-5 \mu \mathrm{m})]$. Lasers for Measurements and Information Transfer: Intern. Conf.: Proc. SPIE. Vol. 4680: 188-194.

3. Remennyi M.A., Zotova N.V., Karandashev S.A., Matveev B.A., Stus’ N.M., Talalakin G.N. 2003. Low voltage episide down bonded mid-IR diode optopairs for gas sensing in the 3,3-4,3 $\mu \mathrm{m}$ spectral range. [Low voltage episide down bonded mid-IR diode optopairs for gas sensing in the 3,3-4,3 $\mu \mathrm{m}$ spectral range]. Sensors \& Actuatoators B: ChemicalVol. 91. № 1-3: 256-261.

4. Zotova N.V., Ilyinskaya N.D., Karandashev S.A., Matveev B.A., Remennyi M.A., Stus' N.M. 2008. Ystochnyky spontannoho yzluchenyia na osnove arsenyda yndyia [Sources of spontaneous emission based on indium arsenide]. Physics and technology of semiconductors. T.42. № 4: 641-657.

5. Kabatsiy V. N. 2009. Obъетпые optycheskye pokrutyia yz khalkohenydnukh stekol dlia poluprovodnykovukh ystochnykov YK-yzluchenyia [Volumetric optical coatings from chalcogenide glasses for semiconductor infrared sources]. Technology and design in electronic equipment. № 4: 38-44.

6. Bletskan DI, Kabatsiy V.M. 2009. Ukraina. Zakhysne, prosvitliuiuche ta fokusuiuche pokryttia na osnovi khalkohenidnoho skla i sposib yoho nanesennia [Ukraine. Protective, illuminating and focussing coating on the basis of chalcogenide glass and its method of application] Pat. № 88565. 10.07.2009.

7. Kabatsiy V.M. 2009. Napivprovidnykove dzherelo infrachervonoho vyprominiuvannia [Semiconductor source of infrared radiation]. Pat. № 8564 Україна. 10.02.2009.

8. Kabatsiy V.M., Bletskan D.I. 2009. Bahatokanalne dzherelo vyprominiuvannia (varianty) [Multichannel source of radiation (options)]. Pat. № 86900 Ukraine. 25.05.2009.

9. Kabatsiy V.M.., Bletskan D.I., Gasinets V.O. 2010. Optychnyi sensor haziv [Optical gas sensor]. Pat. № 89707 Ukraine. 25.02.2010. 